https://doi.org/10.18485/efa.2019.11.ch18

\title{
Poésie et science
}

\author{
Jelena Novaković ${ }^{*}$ \\ Faculté de Philologie, Université de Belgrade
}

L'auteure examine la veine scientifique dans la poésie des $\mathrm{xx}^{\mathrm{e}}$ et $\mathrm{xxI}^{\mathrm{e}}$ siècles, en partant de la théorie de la " poésie scientifique », élaboré par René Ghil, qui met en relief le caractère double de la parole poétique, à la fois « phonétique et idéographique ». Cette théorie est acceptée par les poètes qui trouvent dans la science un modèle esthétique à opposer à l'implication romantique du Moi dans un texte poétique, pour exprimer une vision personnelle et originale du monde moderne, marqué par l'essor de la science (Verhaeren, Brioussov, S. Miličić, Queneau, Ponge, M. Todorović, Luminet, Thériault, etc.).

Mots-clés : poésie, science, poésie scientifique, symbolisme, surréalisme, matérialisme, spiritualisme, égotisme.

\section{Poésie, illustration des concepts scientifiques}

Les rapports entre la poésie et la science ont fait l'objet de réflexion dès l'Antiquité. Dans Phèdre, Platon écrit que «l'espace qui s'étend au-dessus du ciel n'a encore été chanté par aucun des poètes d'ici-bas, et ne sera jamais chanté dignement » (Cité par Luminet 2014 : 45) et, dans Poétique (I, 1447, a - b 17), Aristote affirme qu'il ne suffit pas d'exposer en mètre les sujets qui relèvent de la science pour être poète : «il n'y a rien de commun entre Homère et Empedocle sinon le mètre, si bien qu'il est légitime d'appeler l'un poète et l'autre naturaliste plutôt que poète ${ }^{1} »$.

En cherchant le beau, le discours poétique abonde en ornements et figures de rhétorique, tandis que le discours scientifique, orienté vers le vrai, est clair et méthodique. "Rien n'est plus déplacé que de parler de physique poétiquement, et de prodiguer les figures, les ornements, quand il ne faut que méthode, clarté et

\footnotetext{
*novakovicj@sbb.rs

1 Il en est de même pour Hérodote : « On pourrait mettre l'œuvre d'Hérodote en vers, elle n'en serait pas moins l'histoire» (Poétique, IX, 1451 b 2-3). À la différence du discours historique qui ne rend compte que du particulier, le discours poétique, qui « représente » ou « imite » les exemples généraux de l'action humaine, peut avoir une portée générale et philosophique (Poétique, IX, 1451, b 5-11. Cité dans : Galland-Hallyn \& Hallyn 2001: 181-182).
} 
vérité », écrit Voltaire (Voltaire $1775: 9$ ). Au XIX ${ }^{\mathrm{e}}$ siècle Baudelaire rend compte de cette différence en parodiant l'Art poétique de Boileau : " Des savants, des docteurs les mystères terribles / D'ornements égayés ne sont point susceptibles » (Cité dans Loupâtre - Marchal 2014 : 6). Vers la fin du $\mathrm{xx}^{\mathrm{e}}$ et au début du $\mathrm{xxI}^{\mathrm{e}}$ siècles, Jean-Pierre Luminet constate que la poésie est à première vue la forme d'art la plus éloignée des objets de la science. De ce point de vue, le syntagme « poésie scientifique » apparaît comme un oxymore (Luminet $2014: 45$ ).

Pourtant, la poésie ne manque pas de se référer aux connaissances scientifiques et de les intégrer dans son domaine, si bien que les textes poétiques se présentent aussi comme des illustrations des concepts scientifiques et des paradigmes épistémiques en vigueur à une époque donnée. D'une part, dans certaines périodes de l'histoire littéraire, la science se prête plus facilement à la forme poétique. Dans l'Antiquité dont la science est «à demi poétique » (Bertrand 1897 : 261), Lucrèce unit à la science épicurienne la dimension visionnaire de la poésie, au $\mathrm{xVI}^{\mathrm{e}}$ siècle, la poésie est marquée par le platonisme de Ficin, uni à la tradition de Lucrèce et art et science expriment la même vision orphique de la nature (Lenoble 1969 : 304), tandis qu'au XVIII ${ }^{\mathrm{e}}$ siècle, qui n'est pourtant pas un siècle de poésie, Jacques Delille et André Chénier, qui traitent de science, connaissent un grand succès, ce dernier étant considéré comme le plus grand poète de son temps.

D'autre part, à certaines époques la poésie et la science ont pris des chemins différents. Au XIx ${ }^{\mathrm{e}}$ siècle la science se constitue parfois en contradiction avec la poésie, marquée par les envolées romantiques et par le rejet d'une réalité insatisfaisante, si bien que, comme le remarque Louis Bertrand, « mettre en vers des formules ou des expériences de biologie " se présente comme "souverainement ridicule et anti-poétique » (cité dans Loupâtre - Marchal $2014: 8$ ). On considère que la poésie qui traite de la science s'éteint avec le romantisme, bien que la veine scientifique soit présente, par exemple, chez un Victor Hugo.

Pourtant, c'est justement vers la fin du XIx ${ }^{\mathrm{e}}$ siècle que certains prosateurs et poètes trouvent dans la science un modèle esthétique à opposer à l'implication romantique du Moi de l'auteur dans une œuvre littéraire, comme Gustave Flaubert qui écrit, dans sa lettre à Louise Colet du 6 avril 1853, que « la littérature prendra de plus en plus les allures de la science », ou Émile Zola, qui fonde sa théorie du roman sur les acquisitions de la physiologie et de la sociologie et qui dit que « la grande poésie de ce siècle, c'est la science » (Zola 1906 : 413). À ces deux écrivains, qui se rattachent à la tradition réaliste et naturaliste et qui ont surtout en vue la création romanesque, se joignent Rimbaud, qui plaide, après les parnassiens, dans sa lettre à Izambard du 13 mai 1871, pour une « poésie objective » en prêtant à cette expression un sens social et politique et en se considérant lui-même comme un travailleur qui lutte pour édifier une société nouvelle, et Stéphane Mallarmé qui partage l'aspiration flaubertienne à l'impersonnalité et qui tend à cesser d'être Stéphane que tout le monde connaît pour devenir impersonnel, c'est-à-dire 
« une aptitude qu'a l'Univers spirituel à se voir et à se développer, à travers ce que fut moi », comme il l'écrit dans sa lettre à Henri Cazalis du 14 mai 1867.

\section{Le concept de «poésie scientifique »: René Ghil et ses descendants}

Si on a en vue l'aspiration symboliste à l'impersonnalité, il n'est pas étonnant que ce soit justement au sein du mouvement symboliste qu'est né le concept de "poésie scientifique », mais seulement au moment où il est à son déclin. Ce concept est lancé par un de ses " dissidents », René Ghil, dans son article « De la poésie scientifique » (1909), où celle-ci est définie comme une poésie qui part des « données de la science, et pour la pensée directrice et pour la technique » et qui « représente toute une doctrine, à développement philosophique, sociologique, et d'éthique, et supporte une métaphysique » (Ghil 2008 : 124-125). Au début grand admirateur de Mallarmé qui a écrit un avant-propos pour son Traité $d u$ verbe (1886), Ghil rompt en 1888 avec son maître en rejetant résolument son idéalisme et en considérant que la poésie symboliste est « de par son essence toute émotive », « égotiste », incapable d'atteindre au sens universel qui est le véritable objectif de la création poétique : "Toute œuvre poétique n'a de valeur qu'autant qu'elle se prolonge en suggestion des lois qui ordonnent et unissent l'Être-total du monde» (Ghil 2008 : 146). Mais il garde des traces du symbolisme, si bien qu'on pourrait dire, surtout si on a en vue l'hétérogénéité de ce mouvement (cf. Novaković 2012 : 370-383), qu'au fond il enrichit la poétique symboliste d'une veine scientifique dont il trouve le point de départ chez Mallarmé lui-même, notamment dans la lettre où celui-ci lui rend hommage « de ne point produire au hasard », en appréciant surtout son « effort d'orchestration écrite » (Ghil $2008: 125)$.

Dans ses réflexions sur la «poésie scientifique », Ghil se réfère aux travaux de Christian Gottlieb Kratzenstein et Hermann von Helmholtz, deux physiciens qui ont démontré que les voyelles doivent être considérées comme des timbres vocaux. " Le langage scientifiquement est musique : Helmholtz a, en effet, démontré que, aux timbres des instruments de musique et aux timbres de la voix, les voyelles sont les mêmes harmoniques : l'instrument de la voix humaine étant une anche à note variable complétée par un résonateur à résonance variable, que sont le palais, les lèvres, les dents, etc. », dit Ghil dans sa réponse à l'enquête de Jules Huret sur l'évolution littéraire (Huret 1891 : 112). Mais, ajoute-t-il, la musique ne fait que « décrire » et « suggérer » et ne peut pas « définir », tandis que le langage " décrit, suggère et définit nettement le sens », si bien qu'il est au-dessus de la musique (Huret 1891 : 112).

Les travaux de ces deux physiciens constituent pour Ghil le point de départ dans l'élaboration de ce qu'il appelle l'« instrumentation verbale », c'est-à-dire 
d'un procédé de la poésie scientifique qui « donne à la parole poétique son sens complet et nécessaire en lui rapportant son primordial élément de phonalité » (Ghil 2008 : 149), qui est le « cri ». La mission du poète est de retrouver cette origine perdue du langage, ce qui n'est pas sans rappeler un des objectifs des surréalistes. La théorie de l' " instrumentation verbale », qui met en relief le caractère double de la parole poétique, a orienté beaucoup de poètes vers des recherches de musique verbale et a influencé les théoriciens postérieurs. L'instrument du poète est le « verbe-vidéogramme » qui exprime « totalement » la pensée et l'émotion, par sa valeur phonétique et idéographique, complétée par le " dessin graphique » et des « intensités colorées ». Cette théorie unit en quelque sorte ce qui est exprimé dans les « Correspondances » de Baudelaire, le « Sonnet des Voyelles » de Rimbaud, l'« Art poétique » de Verlaine et les réflexions de Mallarmé pour lequel « l'œuvre pure implique la disparition élocutoire du poète, qui cède l'initiative aux mots » (Mallarmé 2003 : 211), pour ouvrir la voie au vers libre pratiqué par Émile Verhaeren et théoriquement élaboré par Gustave Kahn.

Aux théories physiques qui se rapportent à la langue se joignent les théories évolutionnistes sur lesquelles repose, et dont elle part, la théorie de la poésie scientifique. Il ne s'agit pas de la théorie évolutionniste de Charles Darwin ou des déductions de Spenser et de Nietzsche, fondées sur la loi de la lutte pour la vie, vue comme le but de l'évolution vitale, et que Ghil rejette. Sa théorie est fondée sur « une loi d'amour procréateur » et une « tendance à l'harmonie » dont est pénétrée la Matière, elle a un aspect « altruiste » (voir Bobillot 2014 : 379-395). « Nonconsciente d'elle-même », la Matière est en évolution constante vers la prise de conscience d'elle-même, produite par la science et mue à travers la sensation et la pensée par l'Amour, pris dans le sens « d'affinité chimique » (Ghil 2008 : 151).

Dans ses examens théoriques, Ghil se propose de résoudre l'antinomie du matérialisme et du spiritualisme, qu'il considère comme un avatar de l'antinomie du bien et du mal, de la lumière et de l'ombre. Son spiritualisme, qui demande de prendre plus de conscience du Tout, est rationnel et immanent à la matière même de l'Univers. Son objectif n'est pas de mettre en vers l'histoire naturelle ${ }^{2}$, mais de parvenir à « une métaphysique scientifique » qui rejette le dualisme symboliste au profit d'une vision moniste du monde qui sera aussi celle du surréalisme et des autres mouvements de l'avant-garde, marqués par la tendance à effacer toutes les frontières et à abolir toutes les antinomies.

Le concept de " poésie scientifique » sera accepté et appliqué par certains chercheurs et historiens littéraires, comme Casimir-Alexandre Fusil, auteur du livre La poésie scientifique de 1750 à nos jours, son élaboration, sa consti-

\footnotetext{
2 «Il me paraîtrait oiseux d'insister davantage sur une définition de la "Poésie scientifique". Nous n'en sommes plus aux heures où des ignares, ou des plaisantins de plus ou moins de probité, demandaient ingénument si nous mettions en vers l'histoire naturelle ! S'il s'en trouve encore, c'est au dam de leur intelligence. » (Ghil 2008 : 149).
} 
tution (1917), ou Albert-Marie Schmidt qui examine La poésie scientifique en France au seizième siècle (1938) ${ }^{3}$. Suivent un nombre de chercheurs, dont Muriel Loupâtre, Hugues Marchal et Michel Pierssens, qui se sont engagés avec d'autres chercheurs, à partir de 2007, dans le projet « Euterpe : la poésie scientifique en France de 1792 à 1939 » pour mettre en lumière les œuvres de cette période dont la science contemporaine constitue le sujet principal. Le résultat de ce travail est l'ouvrage collectif électronique, La Poésie scientifique, de la gloire au déclin, mis en ligne en janvier 2014, auquel nous nous référons à plusieurs reprises dans cette communication.

L’idée d'une «poésie scientifique » qui se propose d'exprimer les rapports de l'homme et de l'univers "selon la science », a attiré certains poètes symbolistes que Ghil mentionne dans son texte et qui ont voulu dépasser " l'égotisme » pour « produire l'âme et l'œuvre complexes de l'homme-social » et pour s'approcher du sens universel. Ce sont, entre autres, le poète belge Émile Verhaeren, notamment celui des Campagnes hallucinées (1893), des Villages illusoires (1895) et des Villes tentaculaires (1895), qui cherche «les énergies nouvelles des campagnes inquiètes, troublées intimement, et du monstrueux et intelligent mécanisme des usines, par les villes, au tragique et occulte trafic des Bourses du monde. - et, hors de l'égotisme, produire l'âme et l'œuvre complexes de l'homme social » (Ghil 2008: 135-136) et qui est pour Ghil le représentant authentique de la " poésie scientifique »; ensuite c'est le poète russe Valéri Brioussov qui proclame « que la poésie doit savoir et penser pour en dire nouvellement son émotion et son lyrisme, et à œuvrer ainsi selon son énergique personnalité » (Ghil 2008 : 159) ; aussi bien que Josip Sibe Miličić ${ }^{4}$, auteur du manifeste du « cosmisme » et du recueil de poésie " cosmique » Le Livre de la Joie (1920), le poète serbe que Ghil mentionne dans Les Dates et les ouvres (1923) comme promoteur de la poésie cosmique. Miličić partageait avec Ghil la croyance dans « une grande poésie de l'amour universel cosmique » (Miličić 1920 : 192), en considérant que la tâche du poète est de rétablir les liens entre l'homme et l'univers.

Parmi les écrivains qui se réfèrent à René Ghil et à sa théorie de la " poésie scientifique », il convient de mentionner quelques autres poètes serbes, tels que le moderniste Stanislav Vinaver ${ }^{5}$, avec son « poème cosmique » Les Gardiens $d u$

\footnotetext{
3 Schmidt définit la poésie scientifique comme une poésie qui cherche à " ordonner en une cosmologie les résultats épars de la philosophie naturelle » (Schmidt $1938: 15$ ). En prêtant à ce terme un sens philosophique, il l'emploie surtout pour les œuvres des poètes du XVI e siècle, telles que le Microcosme de Maurice Scève, l'Uranie de Jacques Peletier, les Météores de Jean-Antoine de Baïf, La Semaine ou Création du monde de Guillaume du Bartas, les Hymnes de Ronsard.

${ }^{4}$ Sibe Miličić et René Ghil ont entamé une correspondance après leur rencontre à Paris, lors de l'Exposition de peinture en 1919. Pour plus de détails sur cette correspondance, qui a duré jusqu'à la mort de Ghil en 1925, voir : Kreho 2016: 247-254.

5 En 1922 Vinaver a publié un court article sur le rapport de Ghil à Sibe Miličić, dans la revue littéraire serbe Misao [La Pensée].
} 
monde (1926), où il utilise des symboles anthropologiques et cosmiques, aussi bien que Miroljub Todorović, fondateur et théoricien du signalisme ${ }^{6}$, mouvement artistique et littéraire de la nouvelle avant-garde, né dans la culture serbe dans les années 1960, pour se propager ensuite sur l'espace yougoslave. Dans Le Manifeste de la science poétique (1968), il vise à affranchir l'esprit de la pure subjectivité qui, selon son opinion, domine la poésie serbe, pour lui rendre possible d'établir la communication avec la structure de l'univers. Il plaide pour une poésie scientifique qui exprime l'état d'esprit dans la civilisation technologique et électronique contemporaine par des expérimentations avec le langage qui impliquent la « visualisation » du texte et par des références aux mathématiques et à différentes sciences naturelles (physique, astrophysique, chimie, biologie, biochimie). Dans ses réflexions théoriques, il mentionne trois sources du signalisme : Émile Verhaeren, glorificateur des hommes de science qui s'efforcent d'élargir le domaine de nos connaissances, René Ghil, qui s'est proposé de créer une poésie scientifique et les futuristes qui célébraient le progrès industriel et technologique. Pourtant, pense-t-il, ils ont tous échoué dans leurs tentatives n'étant pas capables de faire la synthèse de la langue poétique et de la langue scientifique. « Tandis que Verhaeren et les futuristes n'ont accepté que les signes extérieurs de la nouvelle civilisation technologique - avion, automobile, phonographe, lumière électrique, machines comme objets poétiques - René Ghil a trop élargi le cercle des sciences susceptibles de s'associer avec la poésie, en y introduisant la sociologie et l'histoire, si bien qu'il s'est perdu dans les labyrinthes de nombreuses disciplines scientifiques peu apparentées » (Todorović 1978 : 78). (Voir : Novaković 2016: 73-88).

\section{Le surréalisme et ses « dissidents » face à la « poésie scientifique »}

La théorie de Ghil n'a pas passé inaperçue par les surréalistes dont le mouvement est une grande entreprise sur le langage, notamment par André Breton et Louis Aragon, qui apprécient pourtant plutôt la musicalité de ses vers que ses réflexions théoriques. Aragon trouve en Ghil un représentant exemplaire de ce « chant à travers les siècles variable, mais toujours étrange » qu'il appelle bel canto et qui n'est autre que « la poésie, quand elle se met à chanter », ce qui la distingue « de cette poésie sans voix à laquelle on voudrait nous confiner» (Aragon 1947 : 87-91, cité dans : Bobillot : 2012). Dans l'entretien radiophonique avec André Parinaud (1952), Breton déclare qu'il « aimait » René Ghil, « le poète le plus décrié du symbolisme », de façon en quelque sorte « subversive » et « distante ». D’une part, il était impressionné par le « volume musical» avec lequel ses

\footnotetext{
${ }^{6}$ Le mot vient du latin « signum ».
} 
poèmes « déferlaient sur une salle » et qui « dominait tous les autres » et surtout par sa persévérance dans la lutte pour un « art difficile et sacré ». D'autre part, à la fois « contrarié » et «attiré » par l'hermétisme de ses poèmes qui le plongeaient dans « une sorte de nuit verbale, ponctuée de rares étincelles », il rejetait sans réserve ses préoccupations «pseudo-scientifiques » (Breton 1969 : 14). Cette déclaration semble confirmée dans un autre entretien avec Parinaud, publié dans Arts (7 mars 1952) :

Je ne vois pas pourquoi, de si tôt, la science et l'art cesseraient de se regarder en chiens de faïence. Du train où la civilisation est allée, dans une direction qu'après Rousseau et Fourier je tiens pour non nécessaire et même totalement aberrante, je ne vois pas comment pourraient converger les deux routes labyrinthiques de la science et de l'art (Breton 1969 : 293).

À première vue, il semble que le surréalisme, qui s'efforce de mettre en échec la pensée logique et les conventions sociales et morales et de réhabiliter l'irrationnel, est incompatible avec la science. « Je maudis la science, cette sœur jumelle du travail », écrit Aragon dans " Fragments d'une conférence » (Aragon 1925 : 24). Pourtant, l'objectif des surréalistes n'est pas esthétique, mais cognitif. Ils identifient la poésie à la vie même, considérée comme un « cryptogramme $\gg^{7}$ qui contient un message chiffré et qui demande un effort de déchiffrement. Aussi leurs procédés reposent-ils sur certaines bases scientifiques ou pseudo-scientifiques, telle que l'expérimentation à laquelle Aragon attribue une « valeur révolutionnaire » car elle rend possible au matérialisme dialectique de progresser dans la connaissance du monde tel qu'il est en réalité (Aragon 1931: 8). Cette expérimentation se réfère à la psychanalyse, notamment à la méthode freudienne d'associations libres que Breton a eu l'occasion de connaître dès 1916, alors qu'il était mobilisé au centre de neurologie à Saint-Dizier ${ }^{8}$, où il utilisait les acquisitions de cette nouvelle science, encore insuffisamment connue en France. Ce travail lui a découvert les possibilités de pénétrer les secrets des phénomènes psychiques, mais aussi de changer son rapport au monde par la simulation des aliénations mentales, par l'analyse des rêves ou en suivant la dictée de l'inconscient. Dans le Manifeste du surréalisme (1924), Breton « rend grâce » aux découvertes de Freud et, dans Les Vases communicants (1932), il commente La Science

\footnotetext{
${ }^{7}$ « Il se peut que la vie demande à être déchiffrée comme un cryptogramme.» (Breton 1964 : 133).

${ }^{8}$ Il est intéressant de mentionner ici la remarque spirituelle d'Henri Béhar, dans son texte introductif de la revue Mélusine no 27 (2007 : 9), consacrée aux rapports entre le surréalisme et la science, concernant la rencontre possible, «surréaliste», de Breton et d'Aragon devant une table de dissection étant donné que tous les deux avait commencé les études de médecine et étaient affectés dans des centres médicaux. Béhar y fait allusion à la définition célèbre de la beauté par Lautréamont comme " la rencontre fortuite sur une table de dissection d'une machine à coudre et d'un parapluie ", à laquelle les surréalistes se référaient souvent.
} 
des rêves, parue en français en 1926. La théorie de l'écriture automatique, qui est un des fondements de la poétique surréaliste, se présente comme l'application directe de la méthode de Freud à des fins surréalistes, mais elle se réfère aussi aux sciences naturelles, comme le montrent Les Champs magnétiques qui font allusion aux découvertes scientifiques de l'époque, le livre qui devait primitivement s'intituler Les Précipités, ce qui renvoie aux expérimentations chimiques. L'objectif du texte automatique n'est pas esthétique, mais scientifique : il découvre « le fonctionnement réel de la pensée », fonctionnement inconscient, dirigé par le désir tout-puissant qui est repoussé par les conventions sociales et morales. ${ }^{9} \mathrm{De}$ ce point de vue, Le Malaise dans la culture (1930) de Freud ${ }^{10}$, où le développement individuel de l'homme et le développement de la société sont représentés comme un processus du refoulement des instincts, qui provoque des frustrations et de névroses, apparaît comme une confirmation explicite de leurs idées (voir Novaković 2009 : 80-97).

Pourtant, une différence fondamentale sépare les surréalistes du fondateur de la psychanalyse. En trouvant dans l'éducation et dans l'organisation sociale des sources des affections nerveuses, Freud ne veut que rendre compte des nécessités auxquelles l'homme doit s'adapter, tandis que les surréalistes se proposent de supprimer les contraintes sociales et morales, en s'en prenant aux principes mêmes sur lesquels la société repose, donc de «transformer le monde ». Aussi pourrait-on dire que leurs références psychanalytiques, comme l'analyse des trouvailles que Breton a faites avec Giacometti dans L'Amour fou, ont une valeur plutôt poétique que scientifique. Il s'agit d'une « poésie de la psychanalyse » (Durozoi - Lecherbonnier : 1974, 197), où les références à la science ont la fonction d'ouvrir le champ de l'imaginaire ${ }^{11}$. En refusant d'accepter le concept de « poésie

\footnotetext{
${ }_{9}^{9}$ À propos de l'impact de la psychanalyse sur la création poétique, on peut mentionner aussi Armen Tarpinian, poète, psychothérapeute et essayiste français d'origine arménienne, qui attribue à la poésie une fonction thérapeutique. Comme la psychothérapie, la poésie est pour lui un moyen de délivrance par l'image et la métaphore est un médicament pour l'angoisse qui l'envahit souvent.

${ }^{10}$ Le Malaise dans la culture est un des principaux livres de référence du surréaliste serbe Vane Bor, qui élabore une théorie du désir dans ses textes « Introduction à la métaphysique de l'esprit » (1930) et « Le talent et la culture » (1934), aussi bien que dans sa réponse à l'enquête sur le désir, publiée en 1932 dans la revue Le Surréalisme aujourd'hui et ici, et dont la traduction française paraît dans Mélusine $n^{\circ}$ XXX (2010), consacrée aux surréalistes serbes. En constant que le refoulement du désir a rompu le rapport spontané de l'homme au monde extérieur, il remarque que cette constatation exige « une large interprétation scientifique » (p. 226).

11 Un exemple: le titre du recueil de Breton Clair de Terre, trouvé dans un livre d'astronomie où cette expression indique l'éclairement des objets dans l'espace par la lumière solaire renvoyée par la Terre, pourrait être inspiré également par les vers du sonnet «Quand l'ombre menaça... » de Mallarmé dont Breton fut grand admirateur : « Oui, je sais qu'au lointain de cette nuit, laTerre / Jette d'un grand éclat l'insolite mystère, / Sous les siècles hideux qui l'obscurcissent moins. ». Mais ce titre se présente aussi comme le contraire de l'expression beaucoup plus fréquente « clair de Lune », ce qui transforme cette expression, selon l'interprétation du surréaliste serbe Dušan Matić, en « une métaphore lumineuse qui gagnait la rue, montait aux toits, illuminait en secret la Terre, noire de
} 
scientifique », Breton soumet la psychanalyse, aussi bien que les sciences naturelles auxquelles il se réfère, à l'objectif surréaliste, la science à la poésie. C'est dans ce sens qu'on pourrait comprendre sa déclaration en quelque sorte prophétique qu'« un jour viendra où les sciences, à leur tour, seront abordées dans cet esprit poétique qui semble à première vue leur être si contraire » (Breton 1924 : $151)^{12}$.

Le texte automatique est une forme d'écriture irrationnelle à opposer au rationalisme, que les surréalistes pratiquent aussi dans la création poétique consciente, en trouvant parfois dans des modèles mathématiques, telle que l'équation, une possibilité de libérer le langage de son écorce de conscience et du poids des conventions morales. C'est ainsi que le poème de Benjamin Péret « 26 points à préciser ", dédié à André Masson et faisant partie du recueil Le Grand $\mathrm{Jeu}$ (1928), consiste en vingt-six vers libres (par analogie aux vingt-six lettres dans l'alphabet), composés de phrases et de formules algébriques et se présente comme une sorte de " texte-vidéogramme » où s'unissent le langage poétique et les éléments mathématiques. «Il ne s'agit pas tout à fait d'équations puisque l'égalité n'est jamais marquée mais toujours suggérée - ce qui est peut-être une manière pour le poète de ne pas imposer une définition, mais au contraire d'ouvrir tout un champ de possibles », constate Virginie Duzer dans son article « Le surréalisme en équations », pour conclure :

Mettre le surréalisme en équation ce serait avant tout revenir sur la dimension esthétique des mathématiques [...]. La révolution surréaliste pourrait s'appuyer sur une certaine forme de poésie scientifique. Il s'agirait particulièrement d'une poésie du signe scientifique jouant sur la dimension cryptique du langage. Et cette poésie serait éminemment apte à libérer l'homme (Duzer 204 : 410).

Les expérimentations poétiques, qui jouent des procédures scientifiques en les détournant de manière mi-ludique mi-sérieuse, seront pratiquées par certains « dissidents » du surréalisme, comme Raymond Queneau et Francis Ponge, deux poètes qui, ayant rejeté l'écriture automatique, se réfèrent aux recherches oulipiennes, marquées par un raisonnement mathématique. Dans Petite cosmogonie portative (1950), Queneau décrit l'histoire du monde de ses origines à l'époque moderne, en faisant référence aux nouvelles connaissances scientifiques acquises dans la première moitié $\mathrm{du} \mathrm{xx}^{\mathrm{e}}$ siècle, telles que la théorie de la relativité d'Einstein et la théorie de l'atome primitif de l'abbé Georges Lemaître qui a supposé qu'à l'origine de l'univers aurait pu être une explosion primordiale,

\footnotetext{
haine, s'élevait au ciel, et à peine quarante ans plus tard, devint pour quelques regards d'hommes, pour nous tous, ce qu'on appelle la «réalité», aussi vraie et aussi fantastique que celle de la lune » (Matic 1976 : 97), interprétation qui est tout à fait dans l'esprit de la poétique surréaliste.

${ }_{12}$ Cf. la déclaration de René Ghil en 1924: « Dans cinquante ans le poète sera celui qui commandera à des machines phonétiques. La poésie sera une science ou ne sera plus » (Ghil 2008: 12.
} 
appelée aujourd'hui big-bang. Dans « Le Chant du Styrène » (1959), destiné à accompagner un documentaire cinématographique d'Alain Resnais qui représente une visite des usines de polystyrène Péchiney, le jeu de mots du titre suggère la « polymérisation » (Baillaud $1993: 25-63$ ) du personnage mythique de la sirène ${ }^{13}$, tandis que le recueil oulipien Cent mille milliards de poèmes (1961), qui offre au lecteur la possibilité de combiner des mots et des vers de façon à composer des poèmes en forme de sonnet régulier, se présente, comme nous le lisons dans la préface, comme " une machine à fabriquer des poèmes ». Francis Ponge publie en 1955, dans La Nouvelle Revue Française, le « Texte sur l'électricité », écrit sur commande de la Compagnie d'électricité et destiné à ses architectes, où il recommande à ses lecteurs de rassembler, comme dans un rêve, les connaissances scientifiques récentes, celles d'Einstein et de Poincaré, de Planck, de Bohr et de Heisenberg, pour contribuer à la création des «nouvelles Figures, qui nous permettront de nous confier à la Parole pour parcourir l'Espace courbe, l'Espace non-euclidien. » (Ponge $1955: 15$ ). Une de ces figures est l'électricité, « grande figure métaphysique » (Ponge $1955: 20)$ qui apparaît comme une fée moderne, à la fois princesse, servante et prostitué : «L'électricité, certes, est une princesse, et qu'elle ait le teint du cuivre ne me déplaît pas. [...] Mais, pourtant, cette princesse est aussi une domestique. [...] En effet c'est une prostitué, mais que m'importe, puisque jamais elle ne perd rien de sa distinction, de son éloignement par principe. » (Ponge 1955 : 19-21). Comme dans le cas du " Chant du Styrène » de Queneau, l'objectif pratique cède la place à un effet poétique. Le « Texte sur l'électricité » pourrait être considéré comme un manifeste de la poésie scientifique moderne à partir duquel s'est formée, comme le remarque Jean-Pierre Luminet dans son article « Renaissance de la poésie scientifique (1950-2010) », une nouvelle Pléiade, « avec les étoiles littéraires tels que Raymond Queneau, Charles Dobzynski, Roger Caillois, André Verdet, Maurice Couquiaud ou Jacques Réda » (Luminet 2014 : 45). Et, ajouterionsnous, Jean-Pierre Luminet lui-même.

\section{La « poésie scientifique » aujourd'hui : Jean-Pierre Luminet}

Astrophysicien et poète, Luminet est focalisé, dans sa poésie et dans ses réflexions théoriques, sur les thèmes relatifs à l'astronomie et à la cosmologie, en trouvant une anticipation de la cosmologie relativiste chez Mallarmé, notamment, dans sa lettre à Villiers de l'Isle Adam du 24 septembre 1867, où il écrit : « J'avais, à la faveur d'une grande sensibilité, compris la corrélation intime de

\footnotetext{
13 « Le Chant du Styrène » fait aussi l'objet de recherche de Pierre Lazlo dans son article éponyme, publié dans l'ouvrage collectif mentionné La Poésie scientifique, de la gloire au déclin.
} 
la Poésie avec l'Univers, et pour qu'elle fût pure, conçu le dessein de la sortir du Rêve et du Hasard et de la juxtaposer à la conception de l'Univers. » (Mallarmé 1998 : 724) (cité dans : Luminet $2014: 49)^{14}$.

Luminet distingue quatre périodes de la poésie scientifique qui correspondent aux quatre "révolutions scientifiques »: l'Antiquité gréco-latine de Lucrèce, Aratus ou Manilius, époque où le discours mythique sur l'univers est remplacé par le discours logique (avec Thalès, Démocrite, Pythagore, Platon, Aristote) ; le $\mathrm{xvI}^{\mathrm{e}}$ siècle de la Pléiade, marqué par l'héliocentrisme de Copernic, Kepler et Galilée, qui ont contribué à « minimiser l'importance des affaires terrestres ou humaines » (Luminet 2014: 47); le XvIII ${ }^{\mathrm{e}}$ siècle de Delille, influencé par les Principia (1687) de Newton qui a découvert la loi universelle de la gravitation et le début du $\mathrm{xx}^{\mathrm{e}}$ siècle, marqué par la révolution relativiste et quantique (la théorie de la relativité et de l'espace-temps courbe d'Einstein, les modèles de big-bang de Georges Lemaître, la naissance de la mécanique quantique qui décrit l'infiniment petit). À ces découvertes correspondent certaines œuvres littéraires, comme « Les licous du temps et de l'espace» (1912) du futuriste Marinetti qui « reprend à sa manière les concepts relativistes d'élasticité des distances et des durées », les Gravitations (1925) de Supervielle, la Grande Féerie (1929) de Maurice Maeterlinck, les œuvres de Paul Valéry, Le Dépouillement par l'espace (1966) de Henri Michaux qui y décrit « comment l'espace du dedans renferme autant de gouffres et de lumières lointaines que l'espace galactique, et comment s'y perdre en dérives infinies » (Luminet $2014: 50$ ). Et, ajouterions-nous, une suite de recueils de poésie cosmique des poètes de l'avant-garde serbe, tels que, entre autres, Le Livre de la Joie (1920) de Sibe Miličić, Les Gardiens du monde (1926) de Stanislav Vinaver, Rêverie galactique (1939) de Marko Ristić, qui se réfère à la fois à l'idée de deux infinis de Pascal et à La Volonté de puissance de Nietzsche, Planète du signaliste Miroljub Todorović (1965). En remarquant que chaque révolution scientifique inspire une production poétique abondante, ce qui est le signe que, «même mathématisé et géométrisé », le ciel «n'en garde pas moins une part de son attrait poétique », Luminet envisage la possibilité d'une cinquième révolution, annoncée par « les tentatives actuelles de trouver une théorie unifiée de toute la physique, qui s'appellent théorie des cordes, modèles binaires ou gravitation quantique à boucles » (Luminet $2014:$ 48-49).

Mais, traiter dans un poème un sujet qui relève de la science ne suffit pas pour qualifier ce poème de "scientifique ». À ce sujet, dans son article « Lire la poésie du XVII siècle : enjeux, obstacles et questionnements », Philippe Chométy attire l'attention sur les imprécisions terminologiques quand il s'agit de la

14 «L'espace à soi pareil qu'il s'accroisse ou se nie /Roule dans cet ennui des feux vils pour témoins / Que s'est d'un astre en fête allumé le génie ", lisons-nous dans les derniers vers du sonnet de Mallarmé « Quand l'ombre menaça... », que Luminet cite et analyse dans son examen de la poésie scientifique, en l'envisageant dans le contexte de la cosmologie relativiste. (Luminet 2014 : 49-50). 
poésie dite scientifique : si on considère que l'expression " poésie scientifique » regroupe le plus souvent les poèmes qui ont pour objet de décrire et de célébrer la variété des phénomènes de la nature, on peut se demander en quoi cette poésie est à proprement parler scientifique étant donné qu'elle ne fait que " décrire » et « célébrer » (Chométy 2005 : 297). Mais, ajouterions-nous, on peut se demander aussi si elle est à proprement parler poétique car, tout en prenant thème dans les connaissances de l'époque donnée, comme la définit Ghil (Ghil 1923 : xi), elle devrait aussi satisfaire à l'exigence aristotélicienne d'avoir « une portée générale et philosophique » (Poétique, IX, 1451, b 5-11).

Conscient des apories qu'entraîne le concept même de "poésie scientifique ", qui ressemble à un oxymore, Jean Luminet divise ce genre poétique en deux sous-genres. Le premier est la poésie didactique ou descriptive, qui décrit et glorifie la science dans des « envolées lyriques plaquées sur le jargon du savant » et qui n'exprime qu'un « émerveillement extérieur ». Et si un Du Bartas, un André Chénier, un Jacques Delille ont créé d'authentiques œuvres poétiques, les autres ne font le plus souvent que confirmer le jugement de Platon que l'espace qui s'étend au-dessus du ciel «ne sera jamais chanté dignement ». Le second est la poésie philosophique ou cognitive qui, tout en étant inspirée par la science, est « fondée sur l'émotion fugitive et individuelle » et a « la prétention d'atteindre à l'universel en créant chez le lecteur des résonances émotionnelles ». Cette poésie, « illustrée par des représentants aussi prestigieux que Lucrèce, Dante ou Victor Hugo $»^{15}$, est pour Luminet l'authentique poésie scientifique qu'il cultive luimême. À ces deux genres de poésie scientifique correspondent deux types de poètes : les poètes qui imitent le discours scientifique en traitant des thèmes fournis par la science et les « rêveurs d'univers » qui, « sachant voir au-delà du décor, réinventent le monde ", en complétant d'intuition les matériaux objectifs apportée par les sciences (Luminet $2014:$ 45-46).

Pour les poètes contemporains, ces sciences sont surtout la physique et l'astrophysique. À ce sujet il convient de mentionner Dany Thériault, spécialiste en physique nucléaire, qui travaille comme physicien médical au département de radio-oncologie de l'Hôtel-Dieu de Québec. En 2012 il a publié un livre qui a deux parties. La première, intitulée Harmonies, contient les poèmes visant à découvrir, dans l'esprit d'un symbolisme «scientifique » qu'il a trouvé dans les Charmes de Valéry, des harmonies du monde, souvent cachées derrière un désordre extérieur. La seconde partie est un court texte théorique, intitulé Manifeste post-symboliste, dans lequel il examine son propos d'un point de vue théorique. Conscient que « la société actuelle compte sur la méthode scientifique pour connaître le

\footnotetext{
${ }^{15}$ Dans cette catégorie, Luminet range aussi Maurice Scève, Pierre de Ronsard et les autres poètes français de la Renaissance qui s'étant employés « à faire reculer le « Monstre Ignorance » par la diffusion de la culture antique et scientifique et la rénovation de la langue française », en rappelant que le nom même de « Pléiade » vient de l'astronomie (Luminet 2014 : 43-44).
} 
monde qui l'entoure » et que l'homme du $\mathrm{XXI}^{\mathrm{e}}$ siècle est conditionné à envisager son existence à travers une " démarche pseudoscientifique », il remarque qu'une fois " dépassé le premier degré utilitariste admis, connaître est une émotion, le sentiment simple et gratifiant d'être « égal » à une partie du monde » (Thériault 2012 : 13). Il s'agit, pour parodier Roland Barthes, d'une sorte de plaisir de la science, plaisir esthétique que constitue « l'impression plaisante de cohérence qu'une théorie provoque » et qui actualise la remarque d'Einstein qu' " une fois atteint un certain niveau de technicité, la science et l'art tendent à se fondre dans l'esthétique, la plasticité et la forme $\left.{ }^{16}\right\rangle$ (Thériault $2012: 14$ ).

Ces deux poètes contemporains expriment d'une manière explicite le vrai sens du concept de "poésie scientifique », en précisant la définition de René Ghil : c'est une poésie qui prend des thèmes dans les connaissances scientifiques, mais non pour les décrire ou les glorifier en imitant le discours scientifique, mais pour " rêver » la science, c'est-à-dire pour exprimer une vision personnelle et originale d'un monde marqué par l'essor de la science moderne.

\section{Sources}

Breton 1921 : A. Breton, Les Pas perdus, Paris : Gallimard.

Breton 1964 : A. Breton, Nadja, Paris : Gallimard, [1928].

Breton 1937 : A. Breton, L'Amour fou, Paris : Gallimard.

Breton 1969 : A. Breton, Entretiens (1913-1952), Paris : Gallimard.

Ghil 1923 : R. Ghil, Les Dates et les æuvres. Symbolisme et poésie scientifique, Paris : Les Éditions G. Crès et $\mathrm{C}^{\mathrm{i}}$.

Ghil 2008 : R. Ghil, De la Poésie-Scientifique \& autres écrits. Textes choisis, présentés et annotés par Jean-Pierre Bobillot, Grenoble : ELLUG, Université Stendhal.

Luminet 2014: J.-P. Luminet, « Renaissance de la poésie scientifique (1950-2010) », in:

Muriel Loupâtre, Hugues Marchal et Michel Pierssens (éd), La Poésie scientifique, de la gloire au déclin, ouvrage électronique mis en ligne en janvier 2014 sur le site Épistémocritique, www.epistemocritique.org, 43-65.

Mallarmé 1998: S. Mallarmé, Oeuvres complètes, I, Paris: Gallimard, Bibliothèque de la Pléiade.

Mallarmé 2003: S. Mallarmé, Oeuvres complètes, II, Paris: Gallimard, Bibliothèque de la Pléiade.

Miličić 1920: S. Miličić, « Jedan izvod koji bi mogao da bude Program », Srpski Književni Glasnik, 1920, I, 3.

Péret 1969: B. Péret, CEuvres complètes, I, Paris: Le terrain vague.

Ponge 1955: F. Ponge, «Texte sur l'Électricité », La Nouvelle Revue Française, n 31, 02/07/1955, 1-29.

${ }^{16}$ Paroles d'Einstein rapportées par Archibald Henderson, Durham Morning Herald, 21 août 1955. 
Queneau 1950: R. Queneau, Petite Cosmogonie portative, Paris: Gallimard.

Theriault 2012 : D. Thériault, Harmonies et Manifeste post-symboliste, Québec : Fondation littéraire Fleur de Lys.

Todorović 1978: M. Todorović, Signalizam, Niš: Gradina.

\section{Références bibliographiques}

Aragon 1925 : L. Aragon, « Fragments d'une conférence », La Révolution surréaliste, $\mathrm{n}^{\circ}$ 4, 15 avril 1925, 23-25.

Aragon 1931 : L. Aragon, "Le surréalisme et le devenir révolutionnaire », Le Surréalisme au service de la révolution, no 3, 2-8.

Aragon 1947 : L. Aragon, Chroniques du Bel canto, Paris : Skira, 1947.

Aristote IX : Aristote, Poétique, IX, 1451 b 2-3.

Baillaud 1993: B. Baillaud, « Raymond Queneau, la polymérisation des sirènes », in Denis Hüe (éd.), Sciences, Techniques \& Encyclopédies. Actes du colloque de Montagne-Au-Perche, 28-29 mars 1992, Caen: Paradigme, coll. « Varia », n 8, 25-63.

Bertrand 1897: L. Bertrand, La fin du classicisme et le retour à l'antique dans la seconde moitié du XVIII ${ }^{\mathrm{e}}$ siècle et les premières années du XIX ${ }^{\mathrm{e}}$ en France, Paris: Hachette, 1897.

Bobillot 2012 : J.-P. Bobillot, « L’anté-tradition futuro-lettriste de René Ghil. Instrumentation verbale et Poésie scientifique », Loxias, 36. Mis en ligne le 15 mars 2012, URL : http://revel.unice.fr/loxias/index.html?id=7029.

Bobillot 2014: J.-P. Bobillot, "René Ghil : altruisme et poésie scientifique », in Muriel Louâpre, Hugues Marchal et Michel Pierssens (éd.), La Poésie scientifique, de la gloire au déclin, ouvrage électronique mis en ligne en janvier 2014 sur le site Épistémocritique, www.epistemocritique.org, 379-395.

Bor 1934: Vane Bor, « Le talent et la culture », Danas, 1er avril 1934, No 4, 44-54.

Chométy $2005: \mathrm{Ph}$. Chométy, « Lire la poésie du XVII ${ }^{\mathrm{e}}$ siècle : enjeux, obstacles et questionnements ", in Libertinage et philosophie au XVII ${ }^{\mathrm{e}}$ siècle, Les Libertins et la science, Publications de l'Université Saint-Étienne.

Durozoi \& Lecherbonnier 1974 : Gérard Durozoi \& Bernard Lecherbonnier, André Breton, l'écriture surréaliste, Paris : Librairie Larousse.

Duzer 2014 : Virginie Duzer, "Le surréalisme en équations », in Muriel Loupâtre, Hugues Marchal et Michel Pierssens (éd), La Poésie scientifique, de la gloire au déclin, ouvrage électronique mis en ligne en janvier 2014 sur le site Épistémocritique, www.epistemocritique.org, 399-411.

Galland-Hallyn \& Hallyn 2001: Périne Galland-Hallyn et Ferdinand Hallyn (éd.), Poétiques de la Renaissance: le modèle italien, le monde franco-bourguignon et leur héritage au xvi ${ }^{\mathrm{e}}$ siècle, Genève: Droz.

Huret 1891: J. Huret, Enquête sur l'évolution littéraire, Paris: Bibliothèque Charpentier. Kreho 2016: V. Kreho, « René Ghil et Josip Sibe Miličić : une amitié littéraire », in Jelena Novaković (éd.), Le Symbolisme en son temps et aujourd'hui, Beograd: Faculté de Philologie - Association de coopération culturelle Serbie-France. 
Lenoble 1969: R. Lenoble, Histoire de l'idée de nature, Paris, Albin Michel.

Loupâtre 2014: M. Loupâtre, Hugues Marchal et Michel Pierssens (éd), La Poésie scientifique, de la gloire au déclin, ouvrage électronique mis en ligne en janvier 2014 sur le site Épistémocritique, www.epistemocritique.org

Matic 1976 : D. Matic, André Breton oblique, Fata Morgana.

Novaković 2009 : J. Novaković, Recherches sur le surréalisme, Sremski Karkovci - Novi Sad: Izdavačka knjižarnica Zorana Stojanovića.

Novaković 2012: J. Novaković, « L'impact francophone sur la littérature serbe: le symbolisme français », in Langue, littérature et culture françaises en contexte francophone, (dir. Zvonko Nikodinovski), Skopje, Faculté de Philologie « Blaže Koneski »- Université « Sts Cyrille et Méthode », 370-383.

Novaković 2016: J. Novaković, « La réception d'Émile Verhaeren dans le milieu littéraire serbe“, in Jelena Novaković (éd.), Le Symbolisme en son temps et aujourd'hui, Beograd: Faculté de Philologie - Association de coopération culturelle SerbieFrance.

Schmidt 1938: A.-M. Schmidt, La Poésie scientifique en France au Xvi siècle, Paris: Albin Michel.

Voltaire 1775 : Voltaire, «Aristote », Questions sur l'Encyclopédie, Euvres de M. de Voltaire, 1775, t. XXVI.

Zola 1906: Émile Zola - Euvres critiques, II, Paris : Eugène Fasquelle.

Јелена Новаковић

\section{Поезија и наука}

Посматрана још у античком добу као књижевни жанр који је неспојив са науком, поезија се међутим позива на научна сазнања и често их укључује у свој свет.

У раду се испитују теме и поступци који се позивају на науку, у поезији XX et XXI века, која је у знаку веровања у творачку моћ језика. То веровање је у основи теорије о „научној поезији“ коју је Рене Гил дефинисао као поезију која настоји да „изрази односе човека и света према науци“ (« La poésie scientifique », 1909). Заснована на радовима Кристијана Готлиба Краценштајна и Хермана фон Хелмхолца, који су показали да самогласнике треба посматрати као вокалне боје, Гилова теорија истиче двоструко обележје поетске речи која је истовремено „фонетичка“ и „идеографска“, унапређујући у систем Бодлерова „Сагласја“, Рембоове „Самогласнике“, Верленову „Песничку уметност“ и Малармеово препуштање иницијативе речима.

Идеју о „научној поезији“ прихватају песници који у науци налазе поетички модел обезличавања песничког исказа и песниковог сједињавања са универзалним животом, попут Емила Ведрхарена, Валерија Брјусова, Сиба Миличића, као и оних 
који се поигравају научним поступцима, попут надреалисте Бенжамена Переа или, педесетих година прошлог века, надреалистичких „дисидената“, Ремона Кеноа и Франсиса Понжа, а шездесетих Мирољуба Тодоровића, оснивача сигнализма који се позива на Гила и Верхарена. Донекле потиснута у други план у време одвајања књижевности од реалности и њеног затварања у сопствени свет, које достиже врхунац средином и у другој половини XX века, научна поезија данас као да доживљава нови процват. Од њених представника, пажњу привлаче, између осталих, астрофизичар Жан-Пјер Лимине, који поетизује теме из области астрономије и космологије и Дани Теријо, стручњак за нуклеарну физику који у науци налази нови извор лепоте. Ова два песника изражавају прави смисао појма „научне поезије“, прецизирајући Гилову дефиницију: није у питању подражавање научног дискурса кроз обраду тема које пружа наука, него нека врста „сањарења“ о науци кроз изражавање личне и оригиналне визије једног света обележеног напретком модерне науке.

Кључне речи: поезија, наука, научна поезија, симболизам, надреализам, материјализам, спиритуализам, еготизам. 